\title{
Editorial
}

\section{The First Five Years}

Five years ago I founded Integrated ComputerAided Engineering. We published two issues in 1993 and four issues per year during 1994-1997. The six 1993 and 1994 issues were published as Volume One. In my Editorial in the inaugural issue of the journal published in 1993 I wrote "The focus of ICAE is the integration of leading edge and emerging computer technologies for innovative solution of engineering problems. The journal fosters interdisciplinary research and presents a unique forum for innovative computer-aided engineering". During 1993-1997 every issue of the journal was published on schedule in a timely manner. This is an exceptional record among the new scholarly journals.

During this period we published a good number of special issues reflecting the interdisciplinary nature of the journal such as:

- Object-Oriented Manufacturing Systems

- Artificial Intelligence in Manufacturing and Robotics

- Intelligent Information Systems

- Real-Time Intelligent Control Systems

- Integrated Product and Process Data Management

- Faults in Automated Manufacturing

- Massively Parallel Computing

- Intelligent Manufacturing Systems

We shall continue publishing special issues devoted to a CAE topic of general and current interest. Plans are well underway for the following forthcoming special issues:

- Low Power Electronic Systems

- Real-Time Engineering Systems

- Neural Techniques for Industrial Application

- Intelligent Autonomous Vehicles

- Mechatronics and Machine Automation

- Architectural Trends for Image Processing and Machine Vision

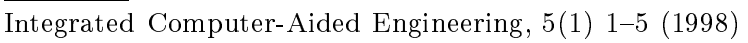

- Industrial Applications of the Wavelet Transforms

A few words about the journal manuscript review process. Our reviewing process is rigorous but fair. The primary criterion for publication in the journal is creativity, originality, and innovation. Each manuscript submitted to the journal is sent to at least three knowledgeable reviewers for review. Manuscripts with clear original contribution are usually processed and accepted in a relatively short period of time. Manuscripts with ambiguous or dubious contribution or poor presentation and English usually take a long time to process. During the first five years we received 316 papers for possible publication in the journal and accepted and published 98 papers which translates into an average acceptance rate of $31 \%$.

In making the final editorial decision an important consideration is the quality of reviews. We seek conscientious reviewers who read the entire manuscript and provide valuable detailed and thoughtful comments. We urge the reviewers to provide constructive comments to improve the content and presentation of the manuscript if it has any merit and original idea. If I am not satisfied with the quality of the reviews additional review or reviews will be solicited.

The manuscripts submitted for possible publication in the journal have been reviewed by members of the Editorial Advisory Board and some 300 other researchers from 27 different countries reflecting the true interdisciplinary and international audience of the journal. Many of these reviewers have agreed in advance to review manuscripts for the journal. The individuals who have reviewed manuscripts for the journal are acknowledged at the end of this editorial. Our apologies for names omitted inadvertently, specially those reviewers whose names were not submitted to me by some of the guest editors.

Starting with this issue, the journal is being published by the Dutch publisher IOS Press. The focus and scope of the journal, however, remains unchanged with the same high standard of pub- 
lication. The editorial decisions are made solely by the same Editor-in-Chief with the help of the members of the Editorial Advisory Board and reviewers. I would be pleased to receive your comments and suggestions. You can send me a fax at +1 614 292-7929 or an email at Adeli.1@osu.edu.

Hojjat Adeli

Editor-in-Chief

Emmanuel D. Adamides, Ecole Polytechnic Federale de Lausanne, Switzerland Sadashiv Adiga, University of California, Berkeley Stan C. Ahalt, The Ohio State University Rudolf Albrecht, University of Innsbruck, Austria Monica Alderighi, IFCTR-CNR, Milan, Italy Cesare Alippi, Polytechnic of Milan, Italy

A. Alpha, University of Manitoba, Canada Shun-ichi Amari, The Institute of Physical and Chemical Research, Japan

Ronald C. Arkin, Georgia Institute of Technology

Giorgio Baccarani, University of Bologna, Italy

Donald J. Bagert, Texas Tech University

Fabrizio Baiardi, University of Pisa, Italy

Carlos Balaguer, University of Carlos III, Madrid, Spain

Felice Balarin, Cadence Berkeley Laboratories

Sanjoy Baruah, University of Vermont

K. Barker, University of Manitoba, Canada

Steven J. Beaty, Cray Computer Corporation, Colorado Springs

George A. Becus, University of Cincinnati

Craig Beebe

Saifallah Benjaafar, University of Minnesota

Azer Bestavros, Boston University

George Betzos

Giacomo M. Bisio, University of Genova, Italy

Mark Boddy, Honeywell Technology Center

Massimo Bombana, ITALTEL, Milan, Italy

Robert Borchelt, University of Wisconsin at Milwaukee

D. Bradshaw, IBM

Bertil A. Brandin, University of Toronto, Canada Jerald R. Brevick, The Ohio State University

M.W. Bright, IBM

S. Brown, University of Tennessee

Alan Burns, University of York

Tiehua Cao, Motorola, Inc.

Jo Dale Carothers, University of Arizona

J. Carruthers, IBM

M. Carson, IBM

Daniele Caviglia, University of Genova, Italy

D. Chandler, IBM
Y. Chehadeh, Pennsylvania State University

Yuh-Min Chen, National Cheng Kung University,

Republic of China

Sunghyun Choi, University of Michigan

Wesley Chu, University of California at Los Angeles

Andrzej Cichocki, The Institute of Physical and

Chemical Research, Japan

J. Cleetus, West Virginia University

Jason Cong

Dario Crosetto, Supercollider Lab, Dallas

Cihan Dagli, University of Missouri at Rolla

Aldo Dagnino, Transmission Technology Institute, Raleigh, North Carolina

Mario Dal Cin, University of Erlangen, Germany

Sivarama Dandamundi, Carleton University, Canada

K. Dash, Pennsylvania State University

Nigel Davis, Lancaster University, United Kingdom

Alessandro De Gloria, University of Genoa, Italy

Giovanni DeMicheli, Stanford University

Didier Demigny, ENSEA, France

Erik de Pablo, Autonomous University of Madrid, Spain

B. Desai, Concordia University, Canada

Chris deSilva, University of Western Australia, Australia

Prasun Dewan, Purdue University

Suzanne W. Dietrich, Arizona State University

Tharam Dillon, La Trobe University, Australia

Lisa Cingiser DiPippo, University of Rhode Island

Kevin Dooley, University of Minnesota

Pamela Drew, University of Science and Technology

Debasish Dutta, University of Michigan

Janet Efstathiou, University of Oxford, United Kingdom

M. Eich, Southern Methodist University

A. Famili, National Research Council, Canada

R. Fauvel, University of Calgary

B. M. Ferretti, University of Pavia, Italy

B. Finch, IBM

Eric Finch, Gensym Corporation

Pamela Fink, Medical Science Systems

Gary W. Fischer, University of Iowa

Phil Fisher

William Fornaciari, Polytechnic of Milan, Italy

Farshad Fotouhi, Wayne State University

David Frank

O. Frieder, George Mason University

K. Fujimura, The Ohio State University

Brian R. Gaines, University of Calgary, Canada

Charles K. Garry, NASA Ames Research Center 
Eric Gayles

Catherine Gebotys, University of Waterloo, Canada

K.M. George, Oklahoma State University

Richard Gerber, University of Maryland

J. Gerrity, IBM

Alain Girault, INRIA Rhone-Alpes, France

Kai Goebel, University of California, Berkeley

Robert Goldman, Honeywell Technology Center

Forouzan Golshani, Arizona State University

E. Gordy, IBM

Marco Gori, University of Siena, Italy

Udo Graefe, National Research Council, Canada

James Graham, University of Louisville

P. Gu, University of Calgary, Canada

Tut San Guan, Queen Mary and Westfield College, United Kingdom

Hans Hansson, Malardalen University College,

Sweden

Jorgen Hansson, University of Skovde, Sweden

Salim Hariri, Syracuse University

Hooshang Hemami, The Ohio State University

M. Hoferek, IBM

L. Hollaar, University of Utah

Larry Holloway, University of Kentucky

W. Timothy Holman

Seongsoo Hong, Seoul National University, Korea

T. Horowitz, Hood College

S.H. Hosseini

David Hsiao

Chua-Huang Huang, The Ohio State University

Ken Hughes, University of the Pacific

Shih-Lin Hung, National Chiao Tung University,

Republic of China

Leslie Interrante, University of Alabama, Huntsville

Shahrukh A. Irani, University of Minnesota

Liliana Ironi, Institute of Numerical Analysis,

Pavia, Italy

Mary Jane Irwin

V. Jagannathan, West Virginia University

Tomasz Janowski, United Nations University, Macau

Kevin Jeffay, University of North Carolina

Agustin Jimenez, Polytechnic University of Madrid, Spain

Niraj K. Jha

Manuel Jimenez

Mathai Joseph, Tata Research Development and Design Centre, India

Sanjay B. Joshi, Pennsylvania State University

Natalia Juristo, Polytechnic University of Madrid, Spain

B. Anthony Kadrvach

Gail E. Kaiser, Columbia University
Vojislav Kecman, University of Auckland, New

Zealand

Wisama Khalil, Laboratoire d'Automatique de Nantes, France

Gary L. Kinzel, The Ohio State University

T. Keefe, Pennsylvania State University

Barney Klamechi, University of Minnesota

Mark Klein, Boeing Computing Services

Yves Kodratoff, University of Paris-South, France

C.K. Koh

Teuvo Kohonen, Helsinki University of Technology, Finland

Jeff Koller

Philip Koopman, Carnegie Mellon University

Mario Koppen, Fraunhofer-Institut IPK Berlin, Germany

R. Korfhage, University of Pittsburgh

David Kortenkamp, NASA Johnson Space Center

Jana Kosecka, University of Pennsylvania

Alexander Kott, Carnegie Group

Gerhard K. Kraetzschmar, Bavarian Research Center for Knowledge-Based Systems

Sergei V. Kulakov, St. Petersburg Academy of Aerospace, Russia

Soundar R.T. Kumara, Pennsylvania State University

Andrew Kusiak, University of Iowa

Rosalba Lamanna de Rocco, University of Simon Bolivar, Venezuela

Tomas Lang

Andrew R. LeBlanc, Clemson University

Dik L. Lee, Hong Kong University of Science and Technology, Hong Kong

Insup Lee, University of Pennsylvania

Mark Lee, University of Wales, United Kingdom

Yann-Hang Lee, University of Florida

Pierre Lefrancois, University of Laval, Canada

Wendy Lehnert, University of Massachusetts

Qing Li, University of Science and Technology

Tilo Lilienblum, University of Magdeburg,

Germany

Jane Liu, University of Illinois at Urbana

Tingyang Liu

Zhi-Qiang Liu, University of Melbourne, Australia

Peter Loborg, Linkoping University

Doug Locke, Lockheed Martin Corporation

Vicente Lopez, Autonomous University of Madrid, Spain

Sam Lor, Griffith University, Australia

Menahem Lowy

G. Luckenbaugh, IBM

Damian Lyons, North American Philips

Corporation

Steven L. Lytinen, DePaul University 
Theo Kangsanant, Royal Melbourne Institute of

Technology, Australia

Charles Meissner, University of Michigan

Lorenzo Mezzalira, Polytechnic of Milan, Italy

Les Miller, Iowa State University

Peter Milligan, The Queen's University of Belfast, United Kingdom

Sang Lyul Min, Seoul National University, Korea

Jack Minker, University of Maryland

Toshimi Minoura, Oregon State University

Christian Mittasch, Freiberg University of

Technology and Mining, Germany

Federico Montecchi, University of Pavia, Italy

Jose Monteiro, INESC, Lisbon, Portugal

Benoit Montreuil, Laval University, Canada

Bob Morley

George Moschytz, Swiss Federal Institute of

Technology at Zurich, Switzerland

Joe Moze, Hong Kong University of Science and

Technology

Al Mok, University of Texas at Austin

Daniel Mosse, University of Pittsburgh

Mohamed Shahid Mujtaba, Hewlett-Packard

Sarit Mukherjee, University of Nebraska-Lincoln

Ravi Mukkamala, Old Dominion University

Nicola Muscettola, NASA Ames Research Center

Chetana Nagendra

Farid Najm

Wolfgang Nejdl, RWTH Aachen, Germany

Gabor Nemeth, Budapest Technical University,

Hungary

Huy T. Nguyen, Georgia Institute of Technology

I. Olasupo, IBM

Mauro Olivieri, University of Genoa, Italy

Gianni Orlandi, University of Rome "La

Sapienza", Italy

Alfonso Ortega

S. Osowski, Technical University of Warsaw, Poland

S. Pakzad, Pennsylvania State University

Rajendran Panda

Marios Papaefthymiou

Christiaan J.J. Paredis, Carnegie Mellon University

Eros Pasero, Polytechnic of Torino, Italy

Ron J. Patton, University of Hull, U.K.

Massoud Pedram

Huei Peng, University of Michigan

Emil M. Petriu, University of Ottawa, Canada

Marc Pirlot, Polytechnic of Mons, Belgium

Edwige Pissaloux, University of Paris XI, France

Reinhard Posch, Graz University of Technology,

Austria

Alberto Prieto, University of Granada, Spain
Miodrag Ptokonjak

Raj Radjassamy

Giancarlo Raiconi, Italy

Srinivasan Ramaswamy, University of Texas at Austin

Ravi M. Rangan

M. Ranganathan, IBM

Michael L. Recce, University College of London, United Kingdom

Nancy Reed, University of Minnesota

Charles Reilly, University of Central Florida

Naphtel Rishe, Florida International University

Pilar Rodriguez, Autonomous University of Madrid, Spain

Kaushik Roy, Purdue University

Stuart H. Rubin, Central Michigan University

Fabrizio Russo, University of Trieste, Italy

P. Sadayappan, The Ohio State University

Miguel A. Salichs, University of Carlos III, Madrid, Spain

Adelio Salsano, University of Rome-Tor Vergata, Italy

Jose M. Sanchez, ITESM, Mexico

Sachin Sapatnekar

Majid Sarrafzadeh

Nello Scarabottolo, Politechnic of Milan, Italy

Alexander Schill, Dresden University of

Technology, Germany

Herman Schmit

Karsten Schwan, Georgia Institute of Technology

Bettina Schweyer, LLP/CESALP, France

Giacomo R. Sechi, IFCTR-CNR, Milan, Italy

Alex V. Shafarenko, United Kingdom

Michael Shanblatt

Chia Shen, Mitsubishi Electric Research Lab

Mark Shephard, Rensselaer Polytechnic Institute

Chia-Hui Shih, National Cheng Kung University

Heonshik Shin, Seoul National University, Korea

Kang G. Shin, University of Michigan

B. Shirazi, University of Texas at Arlington

Rajiv Shivpuri, The Ohio State University

Cristina Silvano, University of Brescia, Italy

Rajendra Singh

Mukesh Singhal, The Ohio State University

Christopher Smith, University of Minnesota

Richard Smith, Secure Computer Corporation

Yeng Chai Soh, Nanyang Technological University, Singapore

Oleg Sokolsky, Computer Command \& Control Company

Richard M. Soley, Framingham Corporate Center, Massachusetts

Sang Hyuk Son, University of Virginia

Matteo Sonza Reorda, Polytechnic of Torino, Italy 
Rogelio Soto, ITESM, Mexico

N. Soundararjan, The Ohio State University

Ben Spaanenburg, University of Groningen, The Netherlands

Otto Spaniol. Aachen University of Technology, Germany

Richard K. Squier, Georgetown University

Sampalli Srinivas, Dalhouise University, Canada

C. Staton, George Mason University

Burkhard Stiller, ETH Zurich, Switzerland

Douglas A. Stuart, University of Texas at Austin

Bogong Su, City University of New York

Mahesh Subramanyan

Roberto Tagliaferri, University of Salerno, Italy

M. Templeton, Data Integration

Charles Traylor

Akhilesh Tyagi

R. Unbehauen, University of Erlangen-Nuremberg, Germany

Susan D. Urban, Arizona State University

Paul Valckenaers, Catholic University of Leuven, Belgium

Mateo Valero, Polytechnic University of Catalunya,
Spain

V. Ventore, MITRE Corporation

Sarma Vrudhula, University of Arizona

Manjula Waldron, The Ohio State University

Fang Wang, Academia Sinica, China

Sarah Wang, Purdue University

Tony Warwick, Quantum Hous, United Kingdom

Lonnie Welch, University of Texas at Arlington

Andy Wellings, University of York

Land S. Wimberley, Lockheed Corporation

Vic Wolfe, University of Rhode Island

Howard Wong-Toi, Cadence Berkeley Laboratories

Bill Wood, University of California, Berkeley

Jie Wood, Florida Atlantic University

Deyi Xue, University of Calgary, Canada

Roni Yagel, The Ohio State University

C.-H. Yen, Iowa State University

Wang Yi, Uppsala University, Sweden

Sergio Yovine, University of California, Berkeley

Emilio Zapata, University of Malaga, Spain

Bertrand Zavidovique, University of Paris XI,

Orsay, France

Yuan F. Zheng, The Ohio State University 